\title{
The radial electric field as a measure for field penetration of resonant magnetic perturbations
}

\section{Saskia Mordijck}

William \& Mary, smordijck@wm.edu
R. A. Moyer
N. M. Ferraro
M. R. Wade
T. H. Osborne

Follow this and additional works at: https://scholarworks.wm.edu/aspubs

Part of the Physics Commons

\section{Recommended Citation}

Mordijck, Saskia; Moyer, R. A.; Ferraro, N. M.; Wade, M. R.; and Osborne, T. H., The radial electric field as a measure for field penetration of resonant magnetic perturbations (2014). Nuclear Fusion, 54(8). https://www.doi.org/10.1088/0029-5515/54/8/082003

This Article is brought to you for free and open access by the Arts and Sciences at W\&M ScholarWorks. It has been accepted for inclusion in Arts \& Sciences Articles by an authorized administrator of W\&M ScholarWorks. For more information, please contact scholarworks@wm.edu. 


\title{
Letter
}

\section{The radial electric field as a measure for field penetration of resonant magnetic perturbations}

\author{
S. Mordijck ${ }^{1}$, R.A. Moyer ${ }^{2}$, N.M. Ferraro ${ }^{3}$, M.R. Wade ${ }^{3}$ and T.H. Osborne ${ }^{3}$ \\ ${ }^{1}$ Department of Computer Science, College of William and Mary, PO Box 8795, Williamsburg, \\ VA 23187-8795, USA \\ ${ }^{2}$ Center for Energy Research, University of California San Diego, 9500 Gilman Dr., La Jolla, CA \\ 92093-0417, USA \\ ${ }^{3}$ General Atomics, PO Box 85608, San Diego, CA 92186-5608, USA \\ E-mail: mordijck@cs.wm.edu
}

Received 8 January 2014, revised 9 May 2014

Accepted for publication 21 May 2014

Published 18 June 2014

\begin{abstract}
In this paper we introduce a new indirect method for identifying the radial extent of the stochastic layer due to applying resonant magnetic perturbations (RMPs) in H-mode plasmas by measuring the spin-up of the plasma near the separatrix. This spin-up is a predicted consequence of enhanced electron loss, due to magnetic stochastization (Kaveeva et al 2008 Nucl. Fusion 48 075003). We find that in DIII-D H-mode plasmas with $n=3$ RMPs applied for edge localized mode suppression, the stochastic layer is limited to the outer 5\% region in normalized magnetic flux, $\Psi_{\mathrm{N}}$. This is in contrast to vacuum modelling predictions where this layer can penetrate up to $20 \%$ in $\Psi_{\mathrm{N}}$. Theoretical predictions of a stochastic radial electric field, $E_{r}$ component exceed the experimental measurements by about a factor 3 close to the separatrix, suggesting that the outer region of the plasma is weakly stochastic. Linear response calculations with M3D-C1, a resistive two-fluid model, show that in this outer 5\% region, plasma response often reduces the resonant magnetic field components by $67 \%$ or more in comparison with vacuum calculations. These results for DIII-D are in reasonable agreement with results from the MAST tokamak, where the magnetic field perturbation from vacuum field calculations needed to be reduced by $75 \%$ for agreement with experimental measurements of the $\mathrm{x}$-point lobe structures (Kirk et al 2012 Phys. Rev. Lett. 108 255003).
\end{abstract}

Keywords: plasmas, stochastic layer, magnetic perturbations

(Some figures may appear in colour only in the online journal)

\section{Introduction}

Research into the effects of non-axisymmetric magnetic perturbations in magnetically confined plasmas is a rapidly growing field. Understanding how these three-dimensional (3D) perturbations affect confinement and stability plays an important role in tokamaks, stellarators and reversed field pinch (RFP) devices. However, in order to be able to distinguish what is causing these changes, we need to be able to assess the underlying magnetic field structure. Currently, resonant magnetic perturbations (RMPs) have successfully been employed to mitigate, suppress or otherwise alter edge localized modes (ELMs) in several tokamak devices (ASDEX-U [1], DIII-D [2], KSTAR [3] JET [4], MAST [5], NSTX [6]). Some predictions for achieving ELM suppression in existing devices and future devices are based upon vacuum calculations (i.e. absence of plasma response) of the magnetic field topology in the plasma edge, called the Chirikov overlap factor [7]. However, different machines have found that while this criterion might be necessary, it is clearly insufficient by itself to achieve ELM suppression $[1,3,5]$.

A large body of work on plasma response (whether theoretical models or numerical simulations) has indicated that plasma response plays an important role in H-mode tokamaks [8-15]. Recent work by Kirk et al [16] on visualizing the perturbed separatrix as a result of RMPs in H-mode plasmas in MAST, has shown that the resonant fields from vacuum field calculations need to be reduced by $75 \%$ in order to match experimental lobe size observations. MAST results differ from TEXTOR L-mode plasmas, where good agreement between vacuum predictions and experiments was observed [17, 18]. Vacuum calculations have also been used to compare particle 
transport changes on different machines and although there is a clear correlation for each machine, there is a large difference among machines [19]. In order to understand the mechanism of transport due to RMPs, one needs to be able to better determine the magnetic topology at the plasma edge for different plasma conditions and machines.

In this paper we will introduce a method that allows us to quantify the width of the stochastic layer in H-mode plasmas. In a stochastic edge plasma, a region of open field lines with short connection lengths (2-3 times the length of field lines in the scrape-off layer) exists and results in an increase in parallel electron loss towards the divertor plates. This non-ambipolar loss, changes the force balance and thus the radial electric field, $E_{r}$, and results in an acceleration of the toroidal rotation in the main ion direction. However, the force balance also has other components, such as the poloidal rotation and the diamagnetic (pressure gradient) component:

$$
E_{r}=\frac{1}{Z_{i} e n_{i}} \frac{\mathrm{d} P_{i}}{\mathrm{~d} r}+v_{\phi_{i}} B_{\theta}-v_{\theta_{i}} B_{\phi} .
$$

Here $E_{r}$ is the radial electric field, $Z$ is the charge of species $i, n_{i}$ is the main ion density and $P$ is the pressure. $v_{\phi, \theta}$ and $B_{\phi, \theta}$ are the ion velocity and magnetic field in the toroidal $(\phi)$ and poloidal $(\theta)$ directions. The absolute value of the diamagnetic component decreases when RMPs are applied [20], and several devices have observed a reduction in the $E_{r}$ well [21-23]. A second component to explain the increase in $E_{r}$ is the experimentally observed acceleration of the carbon toroidal rotation in the ion direction close to the separatrix. This is in qualitative agreement with theoretical predictions which indicate that when RMPs create a stochastic edge, non-ambipolar parallel electron loss increases and creates a $j \times B$ torque in the ion direction [24,25], thus accelerating the toroidal rotation at the plasma edge and increasing $E_{r}$. All experimental observations related to rotation are based on carbon impurity measurements. Moyer et al [26] study the time evolution of the toroidal rotation and radial electric field at the plasma edge and deeper inside the plasma. The changes in the radial electric field at the plasma edge occur on a much faster time scale than the changes in electron density. The radial electric field on the outside of the $E_{r}$ well increases outside error bars on the time scale it takes to ramp-up the RMP coils $(\sim 25 \mathrm{~ms})$. On the time scale that it takes to suppress ELMs $(\sim 100 \mathrm{~ms})$, the radial electric field increases on the inside of the $E_{r}$ well. The final change in $E_{r}$ is on the time scale of the density pump-out ( $150-200 \mathrm{~ms})$. It is on this time scale that the $E_{r}$ well becomes less deep and it takes the plasma about $\sim 200-300 \mathrm{~ms}$ to settle into a new equilibrium. All the analysis presented in this paper, relies on the plasma having reached the new equilibrium state.

In order to sort out the effects of the diamagnetic component and the spin-up in toroidal rotation, we compare the changes in $E_{r}$ as a function of the inverse pressure scale length, $1 / L_{P_{i}}$. In the steep pedestal region, $E_{r}$ scales mostly with the diamagnetic component of the force balance [27] and any differences between discharges with and without the application of RMPs are a direct consequence of the changes in toroidal rotation. Changes to the poloidal rotation have not been observed in the region of interest (in DIII-D low collisionality RMP H-mode discharges [28]). We find that the changes in $E_{r}$ as a function of $1 / L_{P_{i}}$, are independent of changes in $q_{95}$ and that only the outer $5 \%$ of the plasma region inside the separatrix is affected. This is in strong contrast to vacuum field line tracing, using TRIP3D [29], which shows that the outer $15-20 \%$ of the plasma minor radius should be stochastic. To limit the stochastic edge due to the vacuum fields to the last $5 \%$, one would need to reduce the current in the RMP coils by $67 \%$. This simplified model does not reflect the complex plasma response, which will vary on each rational surface. However, this simplified model is in agreement with plasma response modelling with $\mathrm{M} 3 \mathrm{D}-\mathrm{C} 1$ showing that outside $\Psi_{\mathrm{N}}=0.95$, the resonant components of the magnetic field are reduced by $\sim 67 \%$ in these cases.

\section{Changes in radial electric field}

We focus on the analysis of one experiment on DIII-D consisting of 16 different discharges, where $q_{95}$ was varied from discharge to discharge by changing the plasma current, $I_{p}$. All discharges have an identical setup with the exception of the value of $q_{95}$. These are all low collisionality discharges where $v^{*}=q_{95} R \epsilon^{-3 / 2} \lambda_{e}^{-1} \sim 0.05$ at the top of the pedestal. Here, $R$ is the major radius, $\epsilon(\equiv a / R)$ is the inverse aspect ratio, $a$ is the minor radius, $q_{95}$ is the safety factor at the $95 \%$ flux surface and $\lambda\left(=v_{T_{e}} \tau_{e}\right)$, the product of electron thermal velocity $v_{T_{e}}$ and collision time $\tau_{e}$ is the mean free path for electron collisions. All discharges are executed in the ITER similar shape (ISS), with average triangularity $\langle\delta\rangle \sim 0.53$ and all experiments are perturbed by the same $4 \mathrm{kA}$ RMP coil with $n=3$ even parity, where $n$ is the toroidal mode number. These discharges have a fixed neutral beam heating program, so $\beta$, the normalized plasma pressure, drops $\left(\beta_{\mathrm{N}} \sim 2.5-2.1\right)$ when RMPs are employed at constant neutral beam input power (7 MW) and torque. RMPs only suppress ELMs in the discharges where $q_{95}$ is in the resonant window $q_{95} \sim 3.4-3.5$; outside this window, ELMs are mitigated. Figure 1 shows the time evolution of four representative discharges. If the discharge is in the $q_{95}$ resonant window, ELMs are suppressed when the RMP coils are turned on at $2400 \mathrm{~ms}$, as can be observed from the $\mathrm{D}_{\alpha}$ trace. Changes in the pedestal electron pressure are the result of a reduction in pedestal density as the electron temperature remains fairly constant (figure 1). The rotation at the top of the pedestal slows on a similar time scale as the RMP-coil ramp up time.

We construct a database with profiles analysed by multiple users and calculate the inverse ion pressure scale length, $1 / L_{P_{i}}=\nabla P_{i} / P_{i}$ as a proxy for the diamagnetic term. Note that the inverse ion pressure scale length can be made dimensionless by multiplying it with the minor radius, which is the same for each of these experiments, but allows for a comparison across different machines and plasma conditions. It is a good representation for the changes in the diamagnetic contribution, since the main change in pressure is the result of a change in density when RMPs are applied (and not a change in ion temperature). Next we compare $1 / L_{P_{i}}$ to $E_{r}$ at the same radial locations from $\Psi_{\mathrm{N}}=0.9-1$ at 0.01 intervals. Figure 2 shows the difference between experiments without and with RMPs and with and without ELM suppression when RMPs are applied. The darkest colours are from $\Psi_{\mathrm{N}}=0.9-0.95$ and at these radial locations, the three different categories have similar values for $E_{r}$ as a function of $1 / L_{P_{i}}$. The middle 


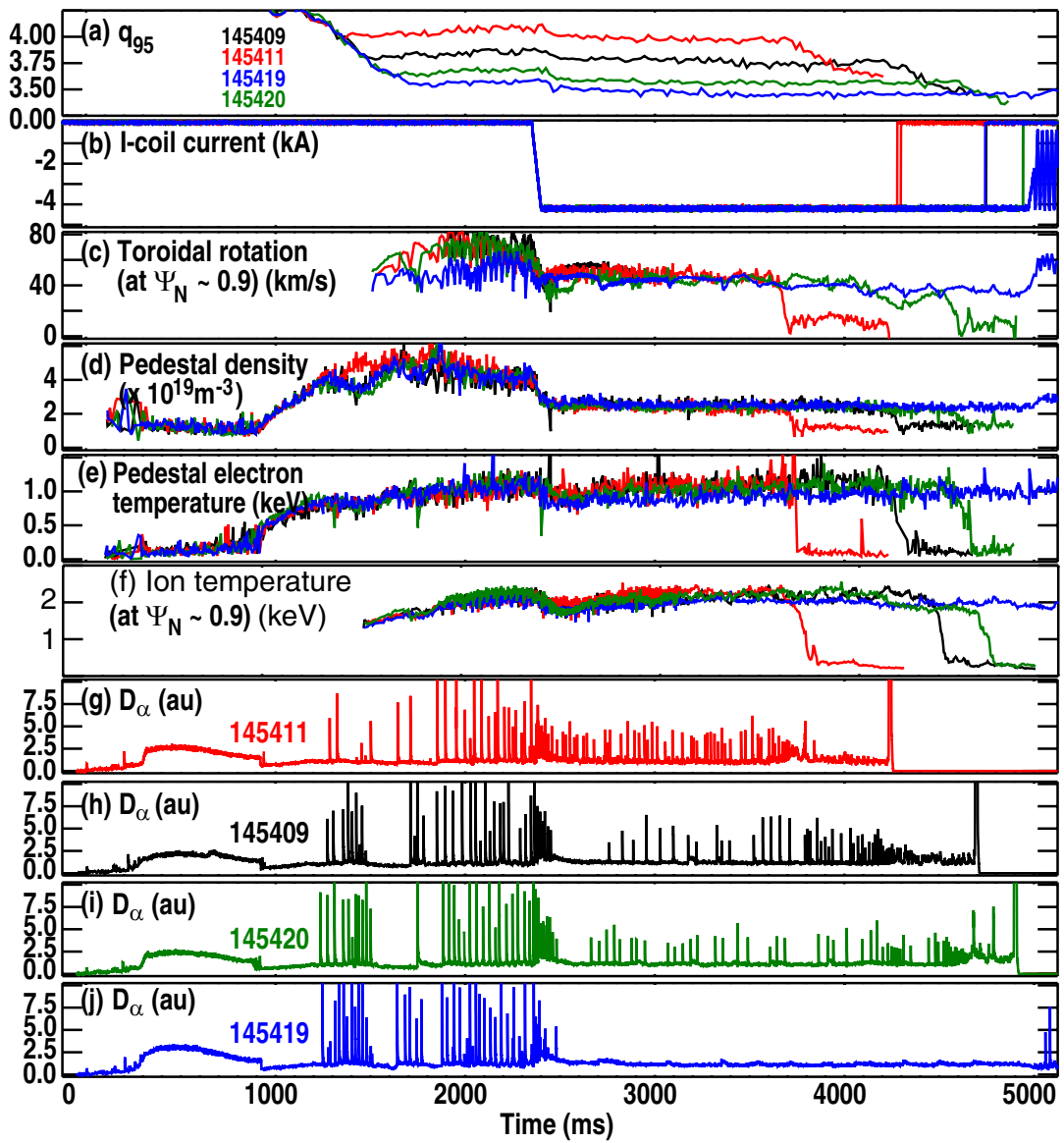

Figure 1. Time traces from 4 representative discharges. The RMP coils are activated $(b)$ at 2400 ms. For all discharges there is a direct reduction in the pedestal density $(d)$. The pedestal electron temperature $(e)$ drops initially but recovers slowly in the discharges. All experiments are performed with constant, but different $q_{95}$ values by changing $I_{\mathrm{p}}$. The toroidal rotation close to the top of the pedestal drops on a similar time scale as the turn-on of the RMP coils. ELMs $\left[\mathrm{D}_{\alpha}\right.$ spikes in $\left.(\mathrm{g})-(\mathrm{j})\right]$ are mitigated in discharges outside the $q_{95}$ resonant window and suppressed inside this window.

colours are from $\Psi_{\mathrm{N}}=0.95-0.97$ and the lightest colours are taken from $\Psi_{\mathrm{N}}=0.97-1$. There is no distinction between ELM suppressed and non-ELM suppressed cases in this data, which suggests that the width of the stochastic layer is not a necessary criterion for ELM suppression. Closest to the separatrix, the cases without RMP application (blue tints) have a lower $E_{r}$ than the cases with RMPs (red/orange and green/yellow colour ranges) for similar $1 / L_{P_{i}}$. The observed change in figure 2 is limited to $\Psi_{\mathrm{N}}>0.95$, which suggests that the region in which stochasticity, or the creation of open field lines, alters plasma parameters is limited to the outer $5 \%$ in $\Psi_{N}$. Additional analysis shows that the poloidal rotation does not change inside this region [28] and thus this increase in $E_{r}$ is the result of a spin-up of the toroidal rotation in the ion direction (figure 3). There is a large spread in the toroidal rotation profile fits, which is a symptom of the uncertainty in the profile fits, which were analysed by multiple scientists using different criteria for obtaining a good fit. As a result, we calculate the average fit for all the profiles with and without RMPs. In the pedestal region there are 4 CER measurement points in these discharges. The measurements outside $\Psi_{\mathrm{N}}=0.95$ show an increase in toroidal rotation on the same time scale as the ramp-up time of the RMP coils. We did not correct for the $3 \mathrm{D}$ deformation of the separatrix in this paper and relied on a $2 \mathrm{D}$ equilibrium reconstruction to map the diagnostic radial measurements. Separate experiments to look at the influence of the 3D deformation of the separatrix and its effects on the toroidal rotation, do not reveal a difference in observed spin-up. This indicates, that the spin-up is the result of the creation of a stochastic edge, not the result in a change of separatrix location.

\section{Comparison to theoretical predictions}

Kaveeva et al [24] derive a theoretical basis for the spin-up of the toroidal rotation as a result of the application of RMPs. This model assumes that in the vicinity of the separatrix there exists a stochastic layer of a certain width. In this layer, the flow of electrons along the open field lines results in a negative current that needs to be offset with a positive radial ion current to maintain ambipolarity. In a strongly perturbed magnetic field, e.g. as imposed by RMPs, a positive radial electric field, $E_{r}^{\mathrm{St}}$, develops:

$$
E_{r}^{\mathrm{St}}=-\frac{T_{e}}{e}\left(\frac{\mathrm{d} \ln n_{e}}{\mathrm{~d} r}+\alpha \frac{\mathrm{d} \ln T_{e}}{\mathrm{~d} r}\right) .
$$

Here, $T_{e}$ is the electron temperature, $n_{e}$ is the electron density and $r$ is the radial coordinate perpendicular to the magnetic flux surfaces. Using this expression for the $E_{r}$, Kaveeva et al [24] derived the change in the toroidal rotation, 


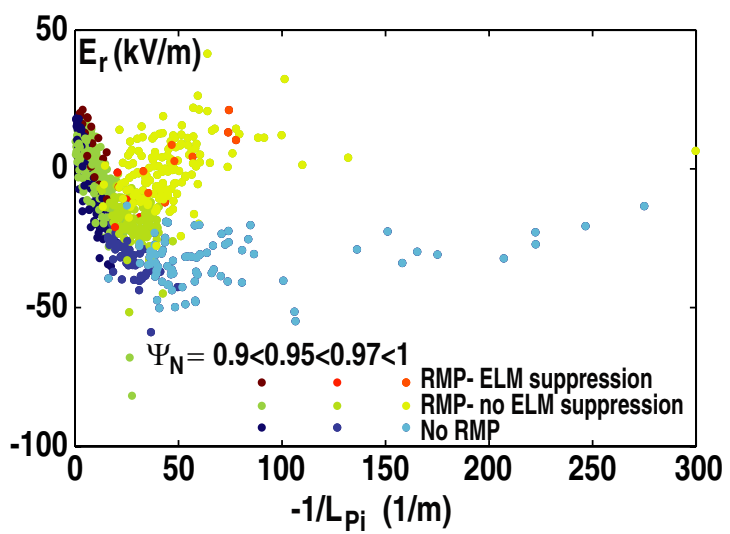

Figure 2. $E_{r}$ versus $1 / L_{P_{i}}$ for 81 independently analysed time slices. The blue colour scheme represents the reference time slices without RMP, the green-yellow scheme is for cases where the RMP coil was on, but ELMs were not suppressed, and the red-orange scheme is for ELM suppressed discharges. The trend from dark to light colours represents the radial location of the data points. $E_{r}$ is more positive outside $\Psi_{\mathrm{N}}=0.95$ when RMPs are applied. There is no difference in the data between the discharges with the RMP coil on or off for $\Psi_{\mathrm{N}}<0.95$.

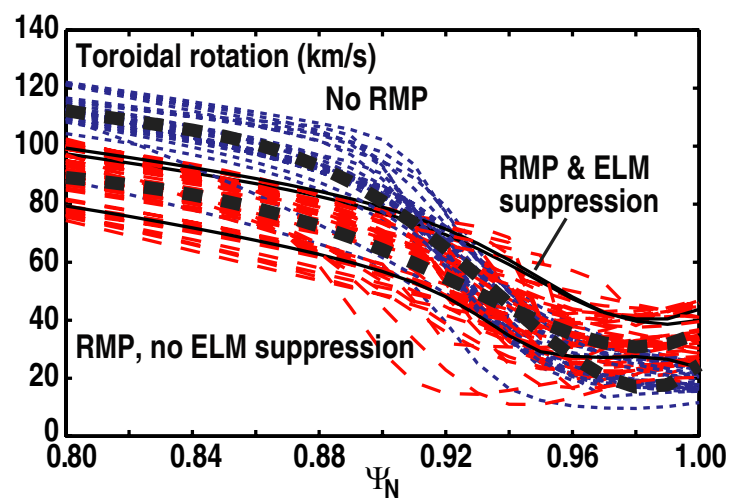

Figure 3. Radial profiles of carbon toroidal rotation for the discharges without RMPs (blue), with RMPs but no ELM suppression (red) and the ELM suppressed discharges with RMPs (black). The solid lines are the polynomial fits to the data and a clear spin-up of the carbon toroidal rotation is observed on average at the plasma edge, whereas a decrease is observed in the core, when RMPs are applied.

$U_{\phi}^{\text {St }}$ as a result of a stochastic layer at the plasma edge:

$U_{\phi}^{\mathrm{St}}=-\frac{T_{e}+T_{i}}{B_{\theta} e} \frac{\mathrm{d} \ln n_{e}}{\mathrm{~d} r}-\alpha \frac{T_{e}}{B_{\theta} e} \frac{\mathrm{d} \ln T_{e}}{\mathrm{~d} r}-k_{T} \frac{T_{i}}{B_{\theta} e} \frac{\mathrm{d} \ln T i}{\mathrm{~d} r}$.

Here, $T_{i}$ is the ion temperature and $\alpha$ and $k_{T}$ are two coefficients $\sim 0.5$ and 1.0 respectively. This theory is a simplification which is valid if the contribution of the poloidal rotation is small in comparison to the contributions of the toroidal rotation and the $E \times B$ drifts. This can be expressed through the parameter $\kappa>1$ [30]. In these experiments, this $\kappa$ ranges from $10^{2}$ at the edge to $10^{5}$ deeper inside.

Figure 4 shows the difference between the theoretical calculation of $U_{\phi}^{\text {St }}$ and the spin-up of the experimental carbon toroidal rotation. The theoretical calculation is 3-5 larger than the experimental observed values and the region where the theoretical calculation predicts a spin-up extends much deeper into the pedestal then experimentally observed. Deeper inside

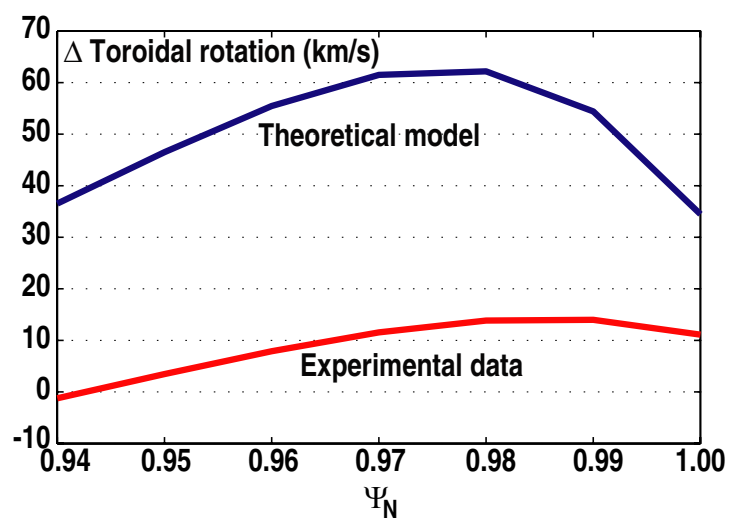

Figure 4. This figure shows the difference in experimental carbon toroidal rotation between discharges with RMPs and those without (red). In the blue curve is the theoretical value for $U_{\phi}^{\mathrm{St}}$. There is large difference between both curves, pointing to the influence of plasma response limiting the stochastic region.

the plasma, the difference in experimental toroidal rotation becomes negative, since the core toroidal rotation slows down when RMPs are applied, whereas $U_{\phi}^{\text {St }}$ remains positive.

Theoretically the spin-up is the result of the non-ambipolar loss of electrons and the concurrent acceleration of the toroidal rotation in the ion direction. In reality, and in contrast to the assumptions of Kaveeva's model, the created stochastic region inside the traditional separatrix does not fully consist of open field lines. Only a fraction of the field lines are open and play a role in identifying the region in which this physics plays a role and might even limit the value of $U_{\phi}^{\text {St }}$. We can calculate the fraction of open field lines using TRIP3D, a field line tracing code, and show that without plasma response, the stochastic layer penetrates deep into the plasma. At $\Psi_{\mathrm{N}}=0.94,50 \%$ of the field lines are still open within 200 toroidal turns of following the field lines (sensitivity studies have shown that more than 200 toroidal turns does not change the field line loss fraction in a significant way) (figure 5). This is in sharp contrast with experimental results from figure 2 that show no change in $E_{r}$ inside $\Psi_{\mathrm{N}}=0.95$. If we assume that the difference between the experimental observations and the theoretical calculations is the result of the edge not being fully stochastic, we can deduce a weighting function that can represent this fraction. To construct this weighting function we have to divide the change in experimental carbon toroidal rotation with $U_{\phi}^{\text {St }}$ (figure 5). This weighting function indicates that plasma response must play a role in limiting the stochastic layer to the outer $5 \%$ of the plasma core, since it is much smaller than the vacuum calculations of the field line loss fraction. In a crude approximation of the plasma response, we reduced the current in the RMP coils by $67 \%$ in the vacuum simulations. This limits the stochastic region to the outer $5 \%$, but the fraction of open field lines at the separatrix is much larger than the predicted weighting function. In the next section, we will discuss these results and compare them to a plasma response model.

\section{Discussion and conclusions}

Previous work on DIII-D shows that the separatrix splits into manifolds and its tangles can be observed on the divertor floor as well as with a soft $\mathrm{X}$-ray camera observing the X-point 


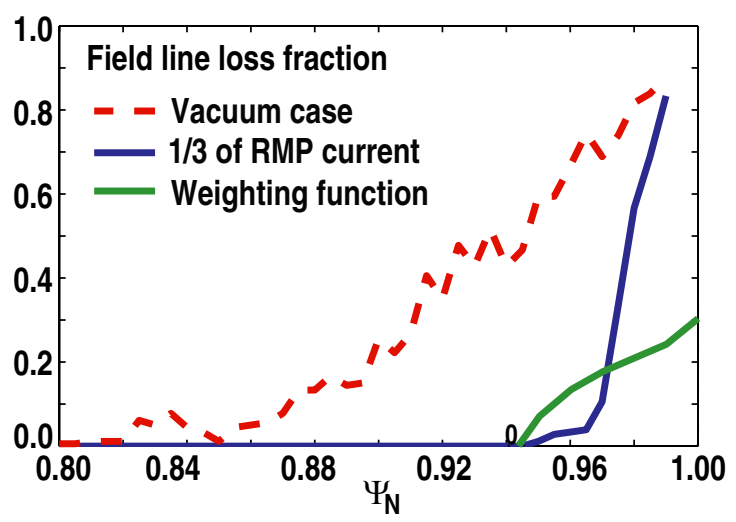

Figure 5. Vacuum calculated field line loss fraction for 1 discharge (145411), with 2 different RMP-coil currents. The red dotted curve is the field line loss fraction based on the experimental input RMP-coil current, whereas the blue solid curve is the field line loss fraction for $1 / 3$ of the experimental input RMP-coil current. The green curve is $\Delta U_{\phi}$ divided by $U_{\phi}^{S} t$ and can be interpreted as a weighting function as a consequence of the plasma edge not being stochastic enough.

region [31,32]. Comparisons with vacuum field line tracing resulted in qualitative agreement, however recent work on MAST visualizing the tangles close to the $\mathrm{X}$-point indicate that the resonant field components obtained from vacuum field calculations need to be reduced by $75 \%$ in order to the match experimental location and size of lobes [16]. The spatial resolution of the MAST measurements is better than those in DIII-D, allowing for a more detailed comparison with modelling. The results on the amount of screening needed at the plasma edge in order to explain the limited width of the stochastic layer with respect to the vacuum calculations presented in the previous section seem to align well with the results from MAST.

In order to better understand why our results indicate that the stochastic region is smaller than predicted by vacuum modelling and theoretical predictions of the spin-up in toroidal rotation, we investigate the role of plasma response using the M3D-c1 code. M3D-c1 is a resistive, two-fluid model that can perform simulations in a diverted, toroidal geometry [14]. In these calculations, the linear solution of the time-independent two-fluid equations are subjected to boundary conditions consistent with the applied fields. This yields perturbed magnetic fields for a single toroidal mode number, in this case, $n=3$. Using a kinetic equilibrium reconstruction [33], figure 6 shows that in the plasma edge $\left(\Psi_{\mathrm{N}}>0.95\right)$ the resonant perturbations are reduced by $67 \%$ in comparison with vacuum field calculations. This reduction in the resonant fields is the well-known screening effect in which plasma rotation (in this case, due to the strong diamagnetic drift in the edge) opposes the opening of magnetic islands [34]. Due to a boundary condition at the separatrix, experimental uncertainties in the fits and equilibrium reconstruction, the one data point in the red curve (figure 6) in the outer 5\% above the vacuum calculations should be taken with a grain of salt. As seen in figure 5, when we apply a simple reduction of the RMP-coil current by $67 \%$ to model the vacuum behaviour of the field lines, we find that stochastic region is now limited to the outer 5\%. However, the total field line loss fraction is still too large. There could be several explanations for this discrepancy close to the separatrix: First and foremost, the

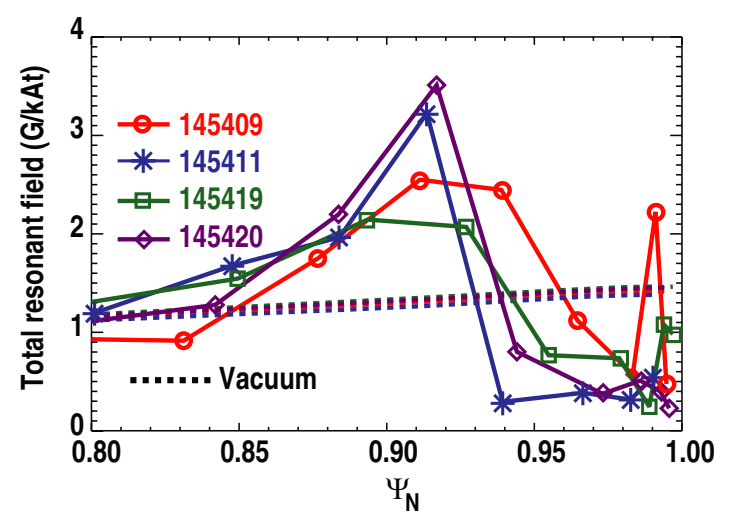

Figure 6. M3D-c1 magnetic field calculations for 4 representative discharges in comparison with the vacuum resonant components. Outside $\Psi_{\mathrm{N}}=0.95$, the plasma response on average reduces the resonant magnetic field by about $67 \%$.

field line loss fraction as a weighting function is a simplification of the complex dynamics that govern edge plasmas; The possibility that only field lines that are lost within a few mean free path lengths should count towards the weighting function (laminar zone versus stochastic region); The influence of scrape-off layer physics so close to the separatrix; The role of turbulence changes to the intrinsic rotation component. Also throughout the paper, we assume that the only change to the toroidal rotation is the result of the creation of a stochastic layer, i.e. that the background momentum transport does not change. A complete accounting of the angular momentum transport should include the effects of NTV and other resonant and non-resonant plasma braking effects. Another reason the spin-up might be less than predicted by Kaveeva et al is that in an $\mathrm{H}$-mode plasma edge, most of the electrons are trapped. This would result in a reduced parallel loss of electrons thus limiting the toroidal spin-up in the main ion direction [35]. In order to fully explain the changes in toroidal rotation, not just the spin-up at the plasma edge, but also the reduction in the plasma core, the full momentum balance needs to be solved, including not only the RMP effects, but also the changes in turbulent transport.

In summary, we find that the stochastic layer formed during application of RMPs is limited to the outer $5 \%$ of the plasma core in DIII-D H-mode plasmas. This is in strong contrast with vacuum field line tracing, where the stochastic region encompasses at least the outer $15 \%$ of the plasma minor radius. When plasma response, i.e. screening in the outer edge due to rotation, is included in the simulations, however, we find quantitative agreement between measured toroidal rotation changes and a simple model. The level of required screening is in good agreement with detailed calculations with the M3D-C1 code.

Our relatively simple analysis technique allows for the estimation of the strength and width of the created stochastic layer in the vicinity of the separatrix as a result of the application of 3D magnetic fields in DIII-D. This analysis technique can be applied for larger numbers of slices of RMP experiments where the $E_{r}$ components and other plasma profiles needed for the simple model were measured [36]. Furthermore, this indirect measurement of the magnetic topology can be applied not only in tokamaks, but also in stellarators and RFP devices. Understanding the underlying 
topology is not only important in order to test plasma response theories, but also to understand changes in confinement.

\section{Acknowledgments}

This work was supported by the US Department of Energy under DE-SC0007880, DE-FG02-07ER54917, DE-FG0295ER54309 and DE-FC02-04ER54698. We would like to thank M.E. Fenstermacher, R. Nazikian and O. Schmitz for sharing their profile analysis. We would like to especially thank P. Peers.

\section{References}

[1] Suttrop W. et al 2011 First observation of edge localized modes mitigation with resonant and nonresonant magnetic perturbations in ASDEX Upgrade Phys. Rev. Lett. 106225004

[2] Evans T.E. et al 2004 Suppression of large edge-localized modes in high-confinement DIII-D plasmas with a stochastic magnetic boundary Phys. Rev. Lett. 92235003

[3] Kim J. et al 2012 ELM control experiments in the KSTAR device Nucl. Fusion 52114011

[4] Liang Y. et al 2007 Active control of type-I edge-localized modes with $n=1$ perturbation fields in the JET tokamak Phys. Rev. Lett. 98265004

[5] Kirk A. et al 2010 Resonant magnetic perturbation experiments on MAST using external and internal coils for ELM control Nucl. Fusion 50034008

[6] Canik J.M. et al 2010 ELM destabilization by externally applied non-axisymmetric magnetic perturbations in NSTX Nucl. Fusion $\mathbf{5 0} 034012$

[7] Fenstermacher M.E. et al 2008 Effect of island overlap on edge localized mode suppression by resonant magnetic perturbations in DIII-D Phys. Plasmas 15056122

[8] Waelbroeck F. 2009 Theory and observations of magnetic islands Nucl. Fusion 49104025

[9] Lanctot M.J. et al 2010 Validation of the linear ideal magnetohydrodynamic model of three-dimensional tokamak equilibria Phys. Plasmas 17030701

[10] Liu Y., Kirk A. and Nardon E. 2010 Full toroidal plasma response to externally applied nonaxisymmetric magnetic fields Phys. Plasmas 17122502

[11] Heyn M.F., Ivanov I.B., Kasilov S.V., Kernbichler W., Joseph I., Moyer R.A. and Runov A.M. 2008 Kinetic estimate of the shielding of resonant magnetic field perturbations by the plasma in DIII-D Nucl. Fusion 48024005

[12] Nardon E., Tamain P., Becoulet M., Huysmans G. and Waelbroeck F.L. 2010 Quasi-linear MHD modelling of $\mathrm{H}$-mode plasma response to resonant magnetic perturbations Nucl. Fusion $\mathbf{5 0} 034002$

[13] Izzo V.A. and Joseph I. 2008 RMP enhanced transport and rotational screening in simulations of DIII-D plasmas $\mathrm{Nucl}$. Fusion 48115004

[14] Ferraro N.M. 2012 Calculations of two-fluid linear response to non-axisymmetric fields in tokamaks Phys. Plasmas 19056105

[15] Park G., Chang C., Jeon Y. and Kim J. 2012 Self-consistent kinetic simulation of RMP-driven transport: collisionality and rotation effects on RMP penetration and transport Proc. 24th IAEA Fusion Energy Conf. (San Diego, CA, 2012) (Vienna: IAEA) www.iaea.org/inis/collection/ NCLCollectionStore/_Public/44/060/44060304.pdf

[16] Kirk A., Harrison J., Liu Y., Nardon E., Chapman I.T. and Denner P. 2012 Observation of lobes near the $X$ point in resonant magnetic perturbation experiments on MAST Phys. Rev. Lett. 108255003

[17] Jakubowski M., Abdullaqev S., Finken K., Lehnen M. and the TEXTOR Team 2005 Heat deposition patterns on the target plates of the dynamic ergodic divertor J. Nucl. Mater. 337-339 176

[18] Jakubowski M., Lehnen M., Finken K., Schmitz O., Abdullaev., Unterberg B., Wolf R. and the TEXTOR Team 2007 Influence of the dynamic ergodic divertor on the heat deposition pattern in TEXTOR at different collisionalities Plasma Phys. Control. Fusion 49 S109

[19] Mordijck S., Moyer R.A., Kirk A., Tamain P., Temple D., McKee G.R. and Nardon E. 2011 Comparison of resonant magnetic perturbation induced particle transport changes in DIII-D and MAST Plasma Phys. Control. Fusion 53122001

[20] Mordijck S. et al 2012 Changes in particle transport as a result of resonant magnetic perturbations in DIII-D Phys. Plasmas 19056503

[21] Tamain P., Kirk A., Nardon E., Dudson B., Hnat B. and the MAST Team 2010 Edge turbulence and flows in the presence of resonant magnetic perturbations on MAST Plasma Phys. Control. Fusion 52075017

[22] Moyer R.A. et al 2006 Edge localized mode control in DIII-D using magnetic perturbation-induced pedestal transport changes Proc. 21st IAEA Fusion Energy Conf. (Chengdu, China, 2006) (Vienna: IAEA) www.iaea.org/inis/collection/NCLCollectionStore/_Public/ 37/098/37098039.pdf

[23] Coenen J.W. et al 2011 Rotation and radial electric field in the plasma edge with resonant magnetic perturbation at TEXTOR Nucl. Fusion 51063030

[24] Kaveeva E., Rozhansky V. and Tendler M. 2008 Interpretation of the observed radial electric field inversion in the TUMAN-3M tokamak during MHD activity Nucl. Fusion 48075003

[25] Kaveeva E. and Rozhansky V. 2012 Screening of resonant magnetic perturbations taking into account a self-consistent electric field Nucl. Fusion 52054011

[26] Moyer R.A. 2014 in preparation

[27] McDermott R.M. et al 2009 Edge radial electric field structure and its connections to H-mode confinement in Alcator C-mod plasmas Phys. Plasmas 16056103

[28] Mordijck S. 2011 Particle transport as a result of resonant magnetic perturbations $P h D$ Thesis University of California, San Diego www.cs.wm.edu/ mordijck/ publications/mordijck_thesis.pdf

[29] Yan L.W. and Evans T.E. 2006 Investigation of background edge thermal transport in ELMing and ELM-suppressed H-modes in DIII-D J. Nucl. Mater. 363-365 723

[30] Rohzansky V., Kaveeva E., Voskoboynikov S., Coster D., Bonnin X. and Schneider R. 2002 Radial electric field in the biasing experiments and effective conductivity in a tokamak Phys. Plasmas 93385

[31] Shafer M.W., Unterberg E.A., Orlov D.M., Evans T.E., Harris J.H., Hillis D.L., Maingi R., Moyer R.A., Nazikian R. and Wingen A. 2012 Experimental imaging of separatrix splitting on DIII-D Nucl. Fusion 52122001

[32] Schmitz O. et al 2008 Aspects of three dimensional transport for ELM control experiments in ITER-similar shape plasmas at low collisionality in DIII-D Plasma Phys. Control. Fusion 50124029

[33] Osborne T.H., Snyder P.B., Burrell K.H., Fenstermacher M.E., Leonard A.W., Moyer R.A., Schaffer M.J. and West W.P. 2008 Edge stability of stationary ELM-suppressed regimes on DIII-D J. Phys.: Conf. Ser. 123012014

[34] Fitzpatrick R. 1993 Interaction of tearing modes with external structures in cylindrical geometry (plasma) Nucl. Fusion 331049

[35] Park G., Chang C.S., Joseph I. and Moyer R.A. 2010 Plasma transport in stochastic magnetic field caused by vacuum resonant magnetic perturbations at diverted tokamak edge Phys. Plasmas 17102503

[36] Viezzer E. et al 2013 High-accuracy characterization of the edge radial electric field at ASDEX Upgrade Nucl. Fusion $\mathbf{5 3} 053005$ 